\title{
EDITORIAL
}

\section{How New Developments Will Change Neuroradiology}

\author{
Claus Zimmer ${ }^{1}$ \\ (c) Springer-Verlag GmbH Germany, part of Springer Nature 2019
}

The motto of this year's conference of the German and Austrian Societies of Neuroradiology (DGNR and ÖGNR) was "The Future of Neuroradiology". For three and a half days neuroradiologists discussed the changes they expect within the next years in the field of diagnostic and interventional neuroradiology. With 1100 participants, this conference is now the biggest neuroradiology event in Europe.

What do we expect to happen in the next years?

In the field of interventional stroke treatment, big steps forward have been made. Within a few years we have succeeded in changing stroke treatment from a bad fate to a high chance of survival, in a lot of cases with excellent outcomes. We have developed and established powerful methods for saving patients' lives and improving their quality of life. It is now a more political task to make this lifesaving treatment fully accessible to large parts of the population in Germany and the rest of the world. Furthermore, a broad range of computer aided methods for analyzing and quantifying brain structure and function has been put on the market. These methods promise to increase the sensitivity for detecting brain abnormalities, which may be invisible to the human eye. They will also add more objectivity to our daily practice, e.g. by automated counting of Multiple Sclerosis (MS) plaques and quantification of brain volume changes over time. Moreover, we expect to gain new knowledge through these techniques, e.g. about the relationship between specific brain functions and anatomical structures.

They will not only change our daily work regarding the interpretation of scans but also on the acquisition side and we will have to ask ourselves whether we really need the numerous T1-weighted (T1w) or T2w magnetic resonance (MR) sequences in the future, which we are using at the moment. I think we don't. Within the next years, synthetic sequences will be generated from a relatively small basic sequence data set, which could potentially replace all our "conventional" sequences from today. A similar development will happen regarding the use of contrast media. I doubt that we will use contrast agents in the near future in the same way as we do today, since new alternative imaging methods will arise.

A main challenge for us physicians will be to redefine our own role. When computers analyze the huge amount of patient data, we have to check the plausibility of all the computer work and we have to communicate the results to our colleagues and to the patient. Our role will be a new one, but this new role will not be less important than it is today.

During our conference, 29 young companies and startups have presented their exciting new developments; however, there are open questions: how can we arrange data exchange without violating data security? How can we integrate these new imaging tools in our clinical routine and really assess their benefit for the patient? How can the costs be refinanced in our healthcare system?

Our conference showed again in an impressive way, how fascinating and diverse our profession is. When we look at the importance of our young colleagues' huge scientific and clinical work, there is no need to worry about the future of neuroradiology.

Conflict of interest C. Zimmer declares that he has no competing interests.

Claus Zimmer

claus.zimmer@tum.de

1 Abteilung für Diagnostische und Interventionelle Neuroradiologie, Klinikum rechts der Isar, TU München, Munich, Germany 